\title{
Physics Issues in the Design of a Recirculating Induction Accelerator for Heavy Ion Fusion
}

\author{
J.J. Barnard, M.A. Newton, L.L Reginato, W.M. Sharp, and S.S. Yu
}

This paper was prepared for submittal to the Intemational Atomic Energy Agency Technical Committee Meeting on Drivers for Inertial Confinement Fusion Oaka, Japan, April 15-19, 1991

April 15, 1991

This is a preprint of a paper intended for publication in a joural or procesding. Since changes may be made before poblieation, this preprint is ande avallable with the understanding that it will not be ciled oc reproduced withoat the perminion of the author.

\section{DISCLAIMER}

This report wis prepared as an account of work sponsored by an agency of the United Statea Government. Neither the United States Government nor any agency th-reol, nor any of their Government. Neither the United States Government nor sny agency thieof, nor any of their bility for the accuracy, completeness, or usefulness of any information, apparatus, product, or prooess disclosed, or represents that its use would not infringe privately owned rights. Reference herein to any specific commercial product, process, or service by trade name, trademark, manufacturer, or otherwise does not necessarily constitute or imply its endorsement, recommanulacturer, or otherwise does nor aecessarily constitute or inply is chency thereof. The views and opinions of authors expressed herein do not necessurily state of reflect those of the United States Government or any agency thereof. 


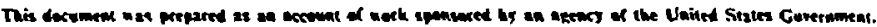

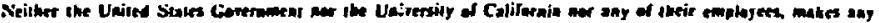

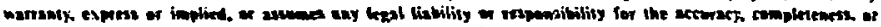

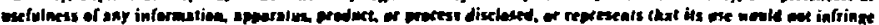

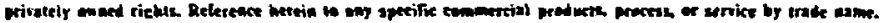

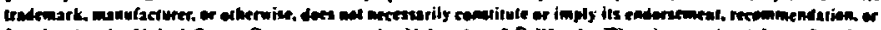

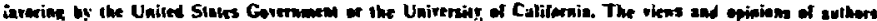

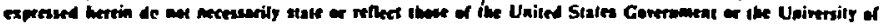

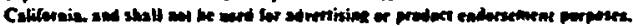


PHYSICS ISSUES IN THE DESIGN OF A RECIRCULATING

INDUCTION ACCELERATOR FOR HEAVY ION FUSION*

\author{
J. J. Barnard, M. A. Newton, L. L. Reginato \\ W. M. Sharp, S. S. Yu \\ Lawrence Livermore National Laboratory, University of California \\ P.O. Box 808, L626, Livermore, Californía, USA 94550
}

*Work performed under the auspices of the U.S. D.O.E. by LLNL under contract W-7405-ENG-48. 
ABSTRACT: $A$ substantial savings in size and cost over a linear machine may be achieved in an induction accelerator in which a heavy ion beam makes many $(<\sim 50)$ passes through one or more circular induction accelerators. We examine how the requirement of high beam quality and the requirement of pulse simultaneity at the target constrain the design of such an accelerator. Some of the issues tbat we have considered include beam interactions with residual gas, beam-beam charge exchange, emittance growth around bends, and beam instabilities. We show some of the interplay between maximization of beam quality and recirculator efficiency, and the minimization of recirculator cost, in arriving at a recirculator design.

\section{INTRODUCTION}

Heavy ion accelerators hold the promise for meeting all of the requirements for an inertial confinement fusion (ICF) reactor for the production of electrical power. These requirements include efficient beam-target coupling, high repetition rate ( $1-10 \mathrm{~Hz}$ ), high reliability, and long stand-off focusing. Previous design studies, have focused on either the linear induction accelerator (e.g. Ref. [1]) or the rf-accelerator/storage ring approach (e.g. Ref. [2]). A substantial cost savings over a linear induction accelerator may be achieved in an induction accelerator in whicb a heavy ion beam makes many ( $\sim 50)$ passes through one or more circularly shaped accelerators. We are examining a few point designs for such an accelerator, consisting of three to four accelerating rings, each of which increments the energy by a factor between 3 and 20 . Since the induction cores used to accelerate the ions are used up to fifty times, the size of the accelerator and the number of cores used can be much smaller, resulting in the possible large cost reduction. However, the introduction of varying magnetic dipole fields, higher peak repetition rate of the induction cells, and the varying voltage pulse format leads to new technical complexities which must be studied before the feasibility of the recirculator can be evaluated. Many of these engineering challenges are addressed in Ref. [3]. In addition, the introduction of bends, of beam manipulations at beam injection and extraction, and the longer residence time of the beam within the accelerator, can lead to degradation of the beam quality.

High beam quality must be maintained throughout the accelerator in order to focus the bearn on a sufficiently small spot at the target. Here beam quality refers to the combination of high current and low emittance. There are a number of processes which degrade the beam quality, by either increasing the transverse emittance, increasing the parallel momentum spread, by removing particles from the beam, or by increasing the bunch length of the beam, and hence lowering the current. In addition the requirement that the individual beams meet simultaneously at the target, impose constraints on the design. In this paper, we discuss some of those processes and constraints, and indicate how they bave impacted the accelerator point designs. In particular we consider the collisional interactions of heavy ions with the background gas, and with other heavy ions in the beam, and with some of the interactions of the beam with the accelerator (in the form of instabilities such as the resistive longitudinal instability). A more complete description of these considerations may be found in Ref. [4].

\section{GENERAL RING ARCHITECTURE CONSIDERATIONS}

Several ideas have been advanced concerning how to place the induction modules, quadrupole focusing magnets and dipole bending magnets into a recirculating ring. One of the primary concerns is to equalize the total path lengths for the different beanlets since it is essential that the beam pulses reach the target simultaneously. In figure 1, four scenarios are advanced all of which satisfy the requirement of equal path lengths. In figure la, the recirculator is shaped like a racetrack consisting of bends, linear portions, and transition regions. In the bends, the beams are stacked vertically insuring equal path lengths. In the linear portion, acceleration takes place through the induction modules. By sending all four beams through one induction core, core volume is minimized, optimizing cost and efficiency. This requires packing the beams into a square array. The space required for changing from square-packed to vertically stacked and any room required for extraction and injection is included in the transition regions between bends and linac sections. By interchanging the top two beams in the bends with the top two beams in the linac, and doing an analogous interchange of the bottom two beams, it is clear that all four path lengths would be identical at all times throughout the accelerator.

In figure $1 \mathrm{~b}$, the racetrack has been replaced by a circular ring, with each lattice period containing a bend and an accelerating cell, in addition to the focusing and defocusing quadrupoles. In this concept (being explored by D. Judd at L.B.L), the four beam pipes appear in cross section to slowly rotate about the longitudinal axis of the accelerator, making a complete rotation during the time of one revolution about the 
major axis of the accelerator. Although at first glance the rotating beam pipes appear quite geometrically complex, Judd has shown that in fact each beam pipe will lie along four separate circles, the center of each being slightly displaced from the center of the accelerator. In addition, each circle is slightly tilted from the horizontal, with the maximum excursions in height of the circles being located in 90 degree intervals around the accelerator (cf. Ref. [5]). In this way al] of the beams have identical path lengths upon one complete lap of the recirculator, although there will be some slight path length differences at intermediate points in the orbit. One clear advantage of this circular approach is that induction cells are available all along the accelerator, so that voltage perturbations may be applied continuously to maintain the appropriate current pulse shape. In the racetrack, long drifts would occur in the bends during which time space chargerill tend to lengthen the pulse. In a racetrack configuration it would be necessary to add corrector modules along the bends in order to preserve the shape of the current pulse. In addition there are no transition regions between stacked and square packed beams which could be a source of beam mismatch, and thus a nassible source of emittance growtl. The disadvantage of the approsch of $1 \mathrm{~b}$ is the uncertainty in the difficulty and cost of aligning the four skewed ring systems.

In figure 1c, the circular approach is adopted to allow conlinuous voltage manipulations, but the beams do not rotate along the mixor axis. Transition regions are provided, in which the beam pipes are switched from a square array to vertical stacking to facillate injection and extraction. To insure equal path lengths, the two upper beams are symmetrically switched so that in one bend region one beam travels in a pipe which is on the inside of the bend, while traveling on the outside of the bend in the other bend region, yjelding equal paths for the two upper beams after one complete circuit, with a similar interchange occuring for the two bottom beams. Here again at internediate times the path lengths will be slightly unequal, but the differences in time amount to be much less than the rise time of the pulse.

In figure 1d, we illustrate an even simpler approach, in which each beam passes through a separate induction core, so that in effect the recirculator ring becomes four separate accelerators stacked on top of each other.

There are some sdvantages to the approach of figure 1d. There is obviously less technical risk in having one core per beam, since there would be no beam-beam interactions in the gaps and no transition regions from square arrays to vertical stacking. Since the beams would be essentially independent, one could even imagine a scenario in which two of the beams could travel on opposite sides of the ring from the other two beams, allowing for a particularly simple double sided illumination of a target in a reactci located at the center of the ring. However, the major disadvantage of separate cores is that the total volume of core material increases. The required area of the longitudinal cross-section of the cylinders which make up the core material (metglas) is proportional to the product of the pulse length and module voltage, which is the same in the two cases. So at first glance the four separate core approach would seem to require four times the volume of metglas. This is offset somewhat by the increased inner radius of the one core scenario, but not completely. The increased volume of metglass in the four core scenario, translates to increased cost and increased core losses.

\section{EMITTANCE GROWTH CONSIDERATIONS}

One of the most serious unresolved issues that confronts the recirculator is the question of how much the normalized emittance will increase on passage of the beam from injector to target. This question is being addressed ou many levels: from crude estimates, through more sophisticated analytic theory to fully 3-dimensional code work, as weil as experimentally. For exanıple, a crude estimate can be made (Ref. [4]) in which it is assumed that alignment errors give rise to betatron oscillations, which are phase mixed, due to non-linearities, and thus give rise to enittance growth. Using the approximation of constant focusing (and a number of other simplifying assumptions) the increased emittance $\Delta \epsilon_{\text {tot }}$ can be written:

$$
\Delta c_{\text {sot }} \cong \sigma \frac{\delta x_{r m i n}^{2}}{L^{2}} n_{\text {lop }} L_{\text {rec }}
$$

Here (with example numbers from the Iligh Energy Ring of the point design), $\sigma \cong .16$ is the depressed tune, the half lattice period $L=300 \mathrm{~cm}$, the number of laps the beam makes in the ring $n_{\text {lop }}=50$, the circumference $L_{\text {rec }}=3.5 \times 10^{5} \mathrm{~cm}$. We fild a $\Delta c_{\text {tos }}=3.2 \times 10^{-3} \mathrm{~cm}$ rat if the rms alignment errors $\delta x_{\mathrm{rms}}=100 \mu$. This compares 10 an average emittance of about $3.8 \times 10^{-3} \mathrm{~cm}$ rad in the design for the High 
Energy Ring. Thus far, estimates of non-linearities and preliminary numerical work have yet to demonstrate that emittance growth will be unmanageable (Ref. [4]), but work in this area is just beginning.

\section{VACUUM CONSIDERATIONS}

The vacuum requirements for the recirculator are somewhat more demanding than for the induction linac approach. The larger residence time of the ion beam in the recirculator by a factor of 10 or \&o, requires an average background gas density that is sinaller by the same factor. Further, the desorbed gasses make a larger contribution to the pumping load in the recirculator than in the linear case.

The background gas and beam deusities are described by fluid equations. See Refs. [4], [6] and references therein for a more complete description. A heuristic derivation is given below which illustrates the basic relationship between background gas and accelerator parameters.

The continuity equation for the background gas (averaged over the volume of the beam pipe) can be written:

$$
\frac{\mathrm{d} n_{g}}{\mathrm{~d} t} \cong q+\left(\eta_{G} \sigma_{i}+\eta_{H} \sigma_{s}\right)\left(V_{b} / V_{p}\right) v_{i} n_{b} n_{g}-\frac{S_{l i n}}{A_{p}} n_{p}
$$

Here, $n$, is the background gas number density, $n_{b}$ is the heavy ion beam number density; $v_{i}$ is the heavy ion velocity; $\sigma_{i}$ is the ionization cross-section of a background gas particle by a heavy ion; $\sigma_{a}$ is the cross section for removing an electron from the heavy ion due to an interaction with a background gas particle; $\eta_{G}$ is the number of molecules desorbed from the pipe inner wall per incident gas molecule; $\eta_{H I}$ is the number of molecules desorbed from the pipe inner wall per incident heavy ion; $S_{\text {in }}=$ linear pump strength (dimensions unit volume per unit time per unit distance); $A_{p}=$ cross-sectional ares of pipe $=\pi r_{p}^{2}$, where $r_{p}=$ pipe radius; $q$ is the intrinsic outgassing .rate per unit distance along the accelerator. Note that $q=2 Q_{0} / r_{p}$ where $Q_{0}$ is the intrinsic outgassing rate per unit surface area; $V_{b}$ the total volume of the beam $=\pi a^{2} v_{i} t_{p}$, where $a=$ beam radius, $t_{p}=$ pulse duration; $V_{p i p e}=$ volume of the pipe $=\pi r_{p}^{2} L_{\text {rec }}$ where $L_{\text {rec }}=$ the circumference of the recirculator.

The beam number density is described by an analogous equation:

$$
\frac{\mathrm{d} n_{b}}{\mathrm{~d} t}=-\sigma_{c} v_{i} n_{b} n_{g}-\sigma_{c e} v_{c m} n_{b}^{2}
$$

Here $\sigma_{c e}$ is the cross-section for a heavy ion to change charge siates due to an interaction with another heavy ion, and $v_{c m}$ is the rms velocity of the heavy ions in the beam frame.

The fractional beam loss $I_{c e}$ from charge exchange alone is:

$$
\begin{gathered}
x_{c e}=\sigma_{c e} n_{i} v_{c m} \Delta t \\
\simeq .025\left(\frac{A}{200}\right)^{.62}\left(\frac{\epsilon_{N}}{.001 \mathrm{~cm} \mathrm{rad}}\right)^{2.24}\left(\frac{Q_{b}}{100 \mu \mathrm{C}}\right)\left(\frac{7 \mathrm{~cm}}{\mathrm{r}_{\mathrm{p}}}\right)^{4.24}\left(\frac{\eta_{p}}{2}\right)^{4.24}\left(\frac{30 \mathrm{~m}}{l_{b}}\right)\left(\frac{\Delta t}{3 \mathrm{~ms}}\right)^{.62}
\end{gathered}
$$

Here we have assumed $\sigma_{c t} \cong 2.1 \times 10^{-16}\left(E_{\mathrm{cm}} / 10 \mathrm{keV}\right.$ ) ${ }^{62} \mathrm{~cm}^{2}$ (cf. Ref. [7]). Also, $E_{c m}=A m_{H} v_{c m}^{2} / 2$; $v_{c m} \cong \epsilon_{N} c j a ; Q_{b}$ is the charge in each ion buncli, the beam radius $a$ is a fraction $\eta_{p}$ of the pipe radius $r_{p}, l_{b}$ is the bunch length, and $\Delta t$ is the residence time of the bunch within the recirculator.

Note the steep dependence of the charge exchange loss on the pipe radius. The first term in equation 4.2 roughly determines the background gas densily,

$$
n_{g} \cong \frac{x_{\text {otrip }}}{\sigma_{,} n_{\text {lap }} L_{\text {rec }}} \text {. }
$$

Here $x_{a \text { rrip }}$ is the fractional beam loss due to stripping, and $n_{\text {lap }}$ is the number of laps of the recirculator catisfying $n_{\text {lap }} L_{\text {rec }}=v_{i} \Delta t$. Eq. (4.4) indicates the scaling that the required gas density is approximately inversely proportional to the residence time.

Equation (4.1) determines the total pumping required to maintain that gas density. Pumping (third term on the right hand side (RHS) of eq. 4.1) balances the sum of the static gas load from intrinsic gas desorption (first lerm on RHS) and the dynamic gas load from beam induced desorption (second lerm on RHS). Thus, 


$$
S_{\text {lin }} L_{\text {rec }} \cong N_{b} n_{\text {lap }} L_{\text {rec }}\left(\frac{\sigma_{g} Q_{o} A_{s p}}{x_{\text {srip }}}+\frac{\left(\eta_{G} \sigma_{i}+\eta_{H I} \sigma_{a}\right) Q_{b}}{g e t_{r}}\right)
$$

Here $N_{\mathrm{b}}$ is the number of beams; $t_{r}$ is the repetition tine for pulses in the tecirculator, and $A_{a p}=2 \pi r_{p} L_{r e c}$ is the total surface area of a single beam pipe. Eq. 4.5 is approximately true when appropriate averages are made over cross sections and desorption coefficient anl also when the second term on the RHS of eq. 4.1 is not exponentially growing. This condition can be expressed as a condition on $r_{p}$ as:

$$
r_{p}>\sim\left(\frac{\eta_{G} \sigma_{i} Q_{b} n_{\text {lap }}}{\pi q e}\right)^{1 / 2}
$$

Here we have neglected $\eta_{H I} \sigma_{s}$ with respect to $\eta_{G} \sigma_{i}$. From eqs. (4.5) and (4.6) we can see that the total pumping rate decreases as $r_{p}$ decreases (as the intrinsic gas desorption decreases) but then increases with further decrease of the pipe radius as the beam induced desorption begins to take off. As an example, in the High Energy Ring of one point design, $N_{b}=\vdots_{1} n_{\text {lap }}=50, L_{\text {rec }}=3.5 \times 10^{5} \mathrm{~cm}, \sigma_{1} \sim 5 \times 10^{-17}$ $\mathrm{cm}^{2}, Q_{0} \sim 10^{-11}$ torr $1 \mathrm{~s}^{-1} \mathrm{~cm}^{-2}, x_{\text {trip }}=.01, \eta_{G} \sim 5, \sigma_{i} \sim 5 \times 10^{-16} \mathrm{~cm}^{2}, \eta_{H l} \sim .01, Q_{b}=100 \mu \mathrm{C}$, $q e=1.6 \times 10^{-19} \mathrm{C}, t_{r}=1 \mathrm{~s}$, so that $S_{\text {lin }} L_{\text {rec }}=3 \times 10^{6}$ liters $\mathrm{B}^{-1}$, which translates into a pumping cost of $\$ 30 \mathrm{M}$ assuming a unit cost of $3 \mathrm{iO} \mathrm{ss}^{-1}$. The requited background gas density would be then be about $3 \times 10^{-10}$ torr.

\section{INSTABILITY CONSIDERATIONS}

A number of potential beam instabilities are being considered, which must be taken into consideration when designing the recirculator (cf. Fef. $[4]$ and references thereiv). Among them are longitudinal resistive instability, beam-break-up instability, and betatron-orbital resonance instability. As an example, we briefly discuss the longitudinal resistive instability. The maximum head to tail logarithmic gain of a perturbation (Ref. [8])

$$
G=N_{b} R v_{i} l_{b} /(2 g)
$$

Here the perturbation growth is by a factor $e^{G}, N_{b}$ is the number of beams, $R$ is the induction module resistance per unit distance, $g$ is the space charge factor $\cong\left(.5+2 \ln \left[r_{p} / a\right]\right)$ The resistance per unit length $R$ must satisfy the condition, that the module impedance is approximately matched to the load on the module to avoid reflections. This leads to the condition,

$$
R=\frac{f}{N_{b} I_{b}} \frac{\mathrm{d} V}{\mathrm{~d} z}
$$

Here $\mathrm{dV} / \mathrm{d} z$ is the average voltage gradient, and $f$ is the reduction in the matched impedance due to losses in the induction core. ( $f=1$ would imply no core losses and the module load rould be strictly from the beam current alone.) Eq. (4.1) may then be approximately written:

$$
\begin{gathered}
G \cong \frac{f}{2 g n_{\text {lap }}} \frac{\Delta V v_{i}^{2} t_{p}^{2}}{L_{\text {rec }} Q_{b}} \\
\cong 18.5\left(\frac{v_{i}}{.3 c}\right)^{2}\left(\frac{t_{p}}{500 \mathrm{~ns}}\right)^{2}\left(\frac{\Delta V}{9 \mathrm{GeV}}\right)\left(\frac{3 \mathrm{~km}}{L_{\text {rec }}}\right)\left(\frac{50}{n_{\text {lap }}}\right)\left(\frac{100 \mu \mathrm{C}}{Q_{\downarrow}}\right)\left(\frac{1.5}{g}\right)\left(\frac{f}{.41}\right)
\end{gathered}
$$

Here $Q_{b}$ is the total charge per beam, $\Delta V$ is the total accelerating potential drop, $L_{\text {rec }}$ is the total circumference of the recirculator, $n_{\text {lap }}$ is the number of laps the beam makes around the recirculator. Note that we have made use of $d V / d z \cong \Delta V /\left(n_{\text {lap }} L_{r e c}\right)$ and $I_{0}=v_{i} t_{p}$. Eq. (4.3) indicates the potentjal advantage of a recirculator over a linear machine in battling longitudinal instability. In a linear accelerator, the quantity $n_{\text {lap }} L_{\text {rec }}$ should be replaced by the acceleralor lenglh $L_{a}$. Since $L_{a} /\left(n_{\text {lap }} L_{\text {rec }}\right)$ can be $<\sim 1 / 15$, and assuming the other factors are the same, it is evident that in principle recirculators can have lower total gains than linear accelerators. Our point recirculator design relies on a pulse forming network to provide pulses of constant duration, so the length of a pulse near the end of the acceleration sequence, is quile long. The combination of high velocity and long pulse durations, i.e. long bunch lengths, yields larger lougitudinal 
instability gains, than would be achieved if an ideal acceleration schedule were used. Using an acceleration schedule in which the pulse duration varied as a function of time occurred, it would be advartageous lo keep $v_{i} t_{p}$ approximately constant equal to its value at the entrance of the high energy ring. The pulse duration $t_{p}$ would then be about 150 ns at the high energy end rather than 500 ns. This would reduce the gain by a factor of 11 , and in fact would make the totai gain turn out to be about 2, rendering the longitudinal instability fairly benign. Inclusion of finite capacitance (Ref. [9]), the possibility of much lower module resistance when programmable switches are used rather than a pulse forming line, and feedback control schemes, all may be invoked to further control resistive instability.

\section{CONCLUSIONS}

Beam quality issues continue to play a central role in evaluating the feasibility of a recirculator for heavy ion fusion. We have given a few exaniples of how our present understanding of these issues helps constrain the accelerator design.

\section{ACKNOWLEDGEMENTS}

The list of authors represents only a small fraction of the people who have contributed to our recirculator studies at L.L.N.L., upon which this paper relies heavily. Sonie of the participants include A. Brooks, F. Coffield, G. Craig, F. Deadrick, J. DeFord, A. Friedman, L. Griffith, D. Hewett, B. Kirbie, G. Lauer, K. Neil, A. Paul, G. Russell, H. Slay, and J. Wilson from Lawrence Livermore National Laboratory, R. Bangerter, A. Faltens, T. Fessenden, C. Fong, D. Judd, and E. Lee from Lawrence Berkeley Laboratory and T. Godlove from F.M.Technologies, Inc.

\section{REFERENCES}

1. Hovingh, J., Brady, V.O., Faltens, A., Keefe, D., and Lee, E.P. "Heavy-Ion Linear Induction Accelerators as Drivers for Inertial Fusion Power Plants," Fusion Technology, 13, (1988) 255.

2. HIBALL-II: An Improved Conceptual Heavy lon Beam Driven Fusion Reactor Study, KRK-3840, Kernforschungszentrum Karlsruhe (July 1985).

3. Newton, M.A., Barnard, J. J., Reginato, L. L., and Yu, S. S., "Engineering Issues in the Design of a Recirculating Induction Accelerator for Heavy Ion Fusion, "these proceedings.

4. Shay, H.D. (ed.), Heavy Ion Fusion Recirculating Induction Accelerator Report, Lawrence Livermore National Laboratory, in preparation, (1991).

¿ Judd, D. "The Circular Linear Induction Accelerator- An Oxymoron?" HIFAR Note-273 (June, 1990)

6. Barnard, J.J., Yu, S.S., Faltens, A., "Vacuum Requirements for Recirculating Induction Accelerators," submitted to Particle Accelerators (volume dedicated to the Proceedings of the International Symposium on Ifeavy Ion Inertial Fusion, December 3-6, 1990, Monterey, CA) (1991).

7. Melchert, F., Salzborn, E., Hofinan, I., Muller, R.W., and Shevelko, V.P., Nuclear Instruments and Methods in Physics Research A278, (1989) 65.

8. Lee, E.P., "Resistance Driven Bunching Mode of an Accelerator Ion Pulse," Proceedings of the 1981 Linear Accelerator Conference, Los Alamos Report LA-9234-C, (1981) 263.

9. Lee, E.P. and Smith, L. "Asymptotic Analysis of the Longitudinal Instability of a Heavy lon Induction Linac," Proceedings of the 1990 Particle Accelerator Conference, Albuquerque, N.M. September 10-14, 1990 (1990).

\section{Figure Captions}

1. Possible Geometrical Configurations of the Recirculator: (a) Racetrack, with bend, linac, and transition regions. (b) Circular, with beam pipes making one rotation about minor axis in one rotation of major axis. (c) Circular, with acceleration occurring in bend regions; transition, injection and extraction occurring in straight sections. (d) Circular, with each beam having a separsite core (or sharing an oblong core) so that the vertically stacked arrangement remains constant throughout the accelerator. 


\section{Racetrack}

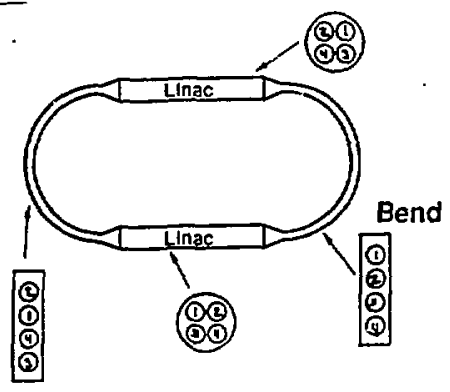

Circular

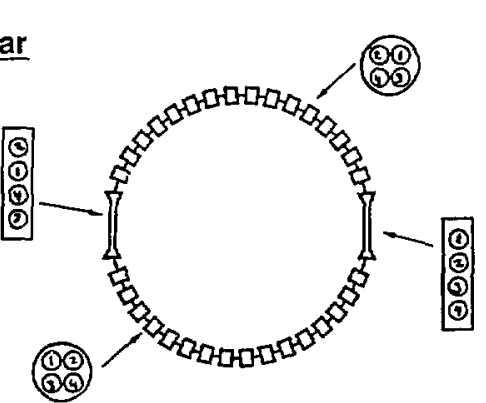

Circular, with azimuthal twist
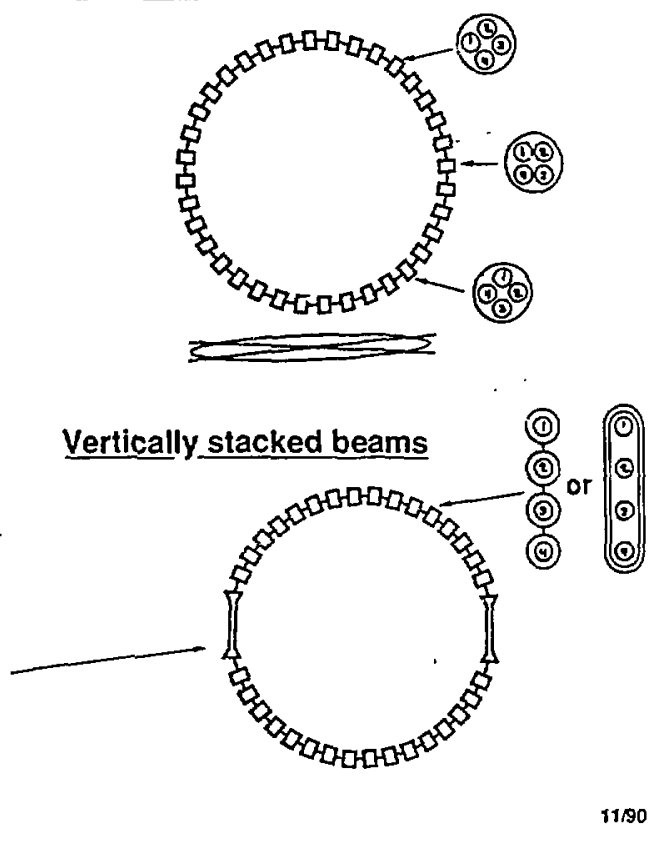

Figure 1. 